\title{
PilG and PilH antagonistically control flagellum-dependent and pili-dependent motility in the phytopathogen Xanthomonas campestris pv. campestris
}

\author{
Yan-Hua Qi, Li Huang, Guo-Fang Liu, Ming Leng and Guang-Tao Lu* D
}

\begin{abstract}
Background: The virulence of the plant pathogen Xanthomonas campestris pv. campestris (Xcc) involves the coordinate expression of many virulence factors, including surface appendages flagellum and type IV pili, which are required for pathogenesis and the colonization of host tissues. Despite many insights gained on the structure and functions played by flagellum and pili in motility, biofilm formation, surface attachment and interactions with bacteriophages, we know little about how these appendages are regulated in Xcc.

Results: Here we present evidence demonstrating the role of two single domain response regulators PilG and PilH in the antagonistic control of flagellum-dependent (swimming) and pili-dependent (swarming) motility. Using informative mutagenesis, we reveal PilG positively regulates swimming motility while and negatively regulating swarming motility. Conversely, PilH negatively regulates swimming behaviour while and positively regulating swarming motility. By transcriptome analyses (RNA-seq and RT-PCR) we confirm these observations as PilG is shown to upregulate many genes involved chemotaxis and flagellar biosynthesis but these similar genes were downregulated by PilH. Co-immunoprecipitation, bacterial two-hybrid and pull-down analyses showed that PilH and PilG were able to interact with district subsets of proteins that potentially account for their regulatory impact. Additionally, we present evidence, using mutagenesis that PilG and PilH are involved in other cellular processes, including chemotaxis and virulence.
\end{abstract}

Conclusions: Taken together, we demonstrate that for the conditions tested PilG and PilH have inverse regulatory effects on flagellum-dependent and pili-dependent motility in Xcc and that this regulatory impact depends on these proteins influences on genes/proteins involved in flagellar biosynthesis and pilus assembly.

Keywords: Virulence factors, Antagonistic control, Motility, PilG and PilH, Xanthomonas

\section{Background}

Xanthomonas campestris is Gram-negative rod-shaped bacteria that causes disease in many plants and is now considered a model organism for the study plant-bacteria interaction [1]. Pathovars of Xanthomonas campestris cause many diseases of agronomic importance throughout the world. One of the most notable of these pathogens is Xanthomonas campestris pathovar campestris $(X c c)$, the

\footnotetext{
*Correspondence: lugt@gxu.edu.cn

State Key Laboratory for Conservation and Utilization of Subtropical Agro-bioresources, College of Life Science and Technology, Guangxi University, 100 Daxue Road, Nanning 530004, Guangxi, China
}

causal agent of black rot of crucifers that affects all cultivated brassicas. The diseases caused by $X c c$ are particularly severe in warm and humid regions, although black rot is also known to have a major impact in regions of temperate climate. $X c c$ is also important as a producer of the extracellular polysaccharide (EPS) xanthan, which is used as an additive in the pharmaceutical and food industries.

The virulence of $X c c$ towards plants depends on several pathogenic factors that include extracellular enzymes (such as cellulase, protease, and amylase), EPS, type three effectors and biofilm formation [2-6]. One

(c) The Author(s). 2020 Open Access This article is distributed under the terms of the Creative Commons Attribution 4.0 International License (http://creativecommons.org/licenses/by/4.0/), which permits unrestricted use, distribution, and 
pathogenic factor of $X c c$ that is gaining more notoriety in virulence is motility. Like most bacteria, Xcc uses a variety of extracellular protein structures to interact with their surrounding environment and drives cellular movement. These extracellular protein structures called flagella and pili contribute cellular movement in the form of 'swimming' and 'swarming', respectively. Additionally, flagellum-dependent and pili-dependent motility are essential to Xcc's ability to attach to host surfaces and to elicit disease. In addition, the flagella and pili are known to be pathogen associated molecular patterns that can induce innate immune responses [7].

Given the importance of flagellum-dependent and pili-dependent motility for $X c c$ survival and ability to cause disease, it is critical that these systems are effectively regulated and controlled. However, despite the numerous studies on bacterial motility in other Gram-negative bacteria, only limited work has been carried out examining the motility regulation in Xcc. The majority of these studies describe putative sensor histidine kinases, putative response regulators or cyclic-di-GMP signalling proteins involved in motility regulation which have included RpfG/RpfC [5], RavS/ RavR [8], ColR/ColS [9], HpaS/HrpG [10], VemR [11] and $\mathrm{VgrR}$ [12]. In majority of cases, deletion or inactivation of the gene encoding these proteins have validated role in flagellum-dependent and pilidependent motility regulation without much follow up work $[1,12]$.

Here we present evidence demonstrating the role of previously uncharacterized single domain response regulators (which we designate PilG and $\mathrm{PilH}$ ) in the regulation of flagellum-dependent and pili-dependent motility in Xcc. Using mutagenesis, we show PilG positively regulates swimming motility while and negatively regulating swarming motility. Conversely, PilH negatively regulates swimming behaviour while and positively regulating swarming motility. Our RNA-seq and RT-PCR experiments confirm these observations as PilG is shown to upregulate many genes involved chemotaxis and flagellar biosynthesis but these similar genes were downregulated by PilH. Additionally, we show that PilH and PilG interact with district subsets of proteins using coimmunoprecipitation, bacterial two-hybrid and pulldown analyses. We also present evidence showing that PilG and PilH are involved in other cellular processes. Overall, this analysis reveals that under the conditions tested PilG and PilH are important in the regulation of motility in Xcc. Interesting the data shows that both proteins have contrasted regulatory effects on flagellumdependent and pili-dependent motility which had not been observed previously.

\section{Results}

PilG and PilH are important for the regulation of pilusdependent and flagellum-dependent motility in Xcc

In our previous work, we isolated large number of $X c c$ mutants from a library constructed using a transposon Tn5gusA5 insertion screen of the Xcc wild-type strain 8004 (genome accession number CP000050) [13]. Two of these mutants with Tn5gusA5 insertions in the open reading frames $X C_{-} 1183$ and $X C_{-} 1184$ were implicated in motility.

Bioinformatic analysis reveals that the $X C_{-} 1183$ gene encodes a 133 amino acid protein that shares a high level of identity to the PilG protein from Pseudomonas aeruginosa, Lysobacter enzymogenes and Acinetobacter baumannii, (e value $=5 \mathrm{e}-77,8 \mathrm{e}-84$ and $8 \mathrm{e}-74$, respectively) (Additional file 1: Figure S1A). Interestingly, the $X C_{-} 1184$ gene also encoded a 120 amino acid protein that had $50 \%$ (e value $=4 \mathrm{e}-45$ ) amino acid homology with $\mathrm{PilH}$ from $P$. aeruginosa, $72 \%$ (e value $=3 \mathrm{e}-66$ ) amino acid homology with PilH from L. enzymogenes, and $52 \%$ (e value $=2 \mathrm{e}-46$ ) amino acid homology with PilH from A. baumannii, respectively (Additional file 1: Figure S1B). We designated the proteins XC_1183 and XC_1184 in Xcc as PilG and PilH for the remainder of the study. Domain analysis using the SMART (Simple Modular Architecture Research Tool) programme (http://smart.embl-heidelberg.de) showed that PilG (XC_ 1183) and PilH (XC_1184) both contained a stand-alone CheY-like REC domain (PilG: 14aa-127aa, PilH: 2aa115aa) (Additional file 1: Figure S1C). Further comparison of PilG and PilH to the well-studied CheY protein domain of Escherichia coli was also carried out. These comparisons showed that PilG shared a $29 \%$ (e value = 2e-20) amino acid homology with CheY, while $\mathrm{PilH}$ showed a $30 \%$ (e value $=3 \mathrm{e}-16)$ amino acid homology (Additional file 1: Figure S1C). Additionally, the previously characterized CheY protein from $X c c$ revealed a $39 \%$ (e value $=6 \mathrm{e}-27)$ amino acid homology with its $E$. coli counterpart (Additional file 1: Figure S1C).

PilG and PilH have been shown to be required for motility in many bacteria, including $P$. aeruginosa $[14,15]$ and Neisseria meningitidis [16] but no roles have been attributed to these proteins in Xcc. In order to explore the function of PilG and PilH in $X c c$, we constructed clean deletions removing $X C_{-} 1183$ and $X_{-} C_{-} 1184$ gene and designating the resulting strains as $\Delta$ pilG and $\Delta$ pilH (see Methods; Additional file 6: Table S1). Simultaneously, these strains were complemented by introducing the plasmid pLAFR3 carrying the $X C_{-}$ 1183 or $X C_{-} 1184$ coding sequence along into the respective mutant. The resulting complemented strains were named CpilG and CpilH (Additional file 6: Table S1). 
Pilus-dependent swarming motility has previously been shown contribute to disease in Xcc [17]. To determine if PilG and PilH are involved in pilus-dependent swarming motility, the constructed strains and wild-type were tested by inoculating on NY plates containing $2 \%$ glucose and $0.6 \%$ agar then incubating at $28^{\circ} \mathrm{C}$ for 3 days (see Methods) [18]. As shown in Fig. 1, $\Delta$ pilG displayed increase $40 \%$ in net migration compared to the wild-type, while showed a $60 \%$ decrease in motility compared to the wild-type (Fig. 1). Importantly, the complementary strains CpilG and $\mathrm{CpilH}$ showed similar motility phenotypes to wild-type (Fig. 1). These findings suggest that PilG negative regulates pilus-dependent swarming in $X c c$, while PilH appears to act as a positive regulator.

$X c c$ has also the ability to swim using its single polar flagellum [19]. To investigate if PilG and PilH are involved in flagellum-dependent swimming motility, the mutants $\Delta$ pilG and $\Delta$ pilH, complemented strains CpilG and $\mathrm{CpilH}$, and wild-type strain were inoculated onto $0.28 \%$ agar plates and incubated for 3 days (see Methods) [18]. As shown in Fig. 1, $\Delta$ pilG showed reduced $90 \%$ compared to wild-type. However, the $\Delta$ pilH deletion strain showed increase of $20 \%$ in swimming motility compared to the wild-type. Importantly, swimming motility of the complementary strains CpilG could be back to half of the wild type and $\mathrm{CpilH}$ showed the restoration of swimming motility phenotypes towards wild-type (Fig. 1). This data suggests that PilG is a positive regulator of flagellumbased swimming but PilH appears to act as a negative regulator which is the converse of what was observed in the pilus-based swarming results.

Taken together, the mutagenesis data described above revealed that PilG and PilH have opposite roles for the regulation of pilus-dependent swarming and flagellumdependent swimming in $X c c$.
A
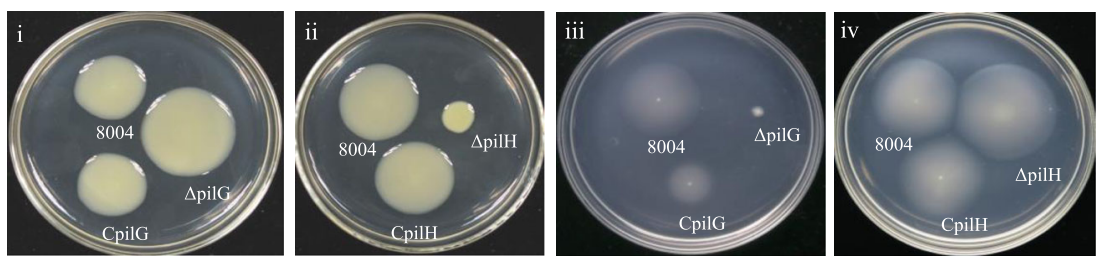

B

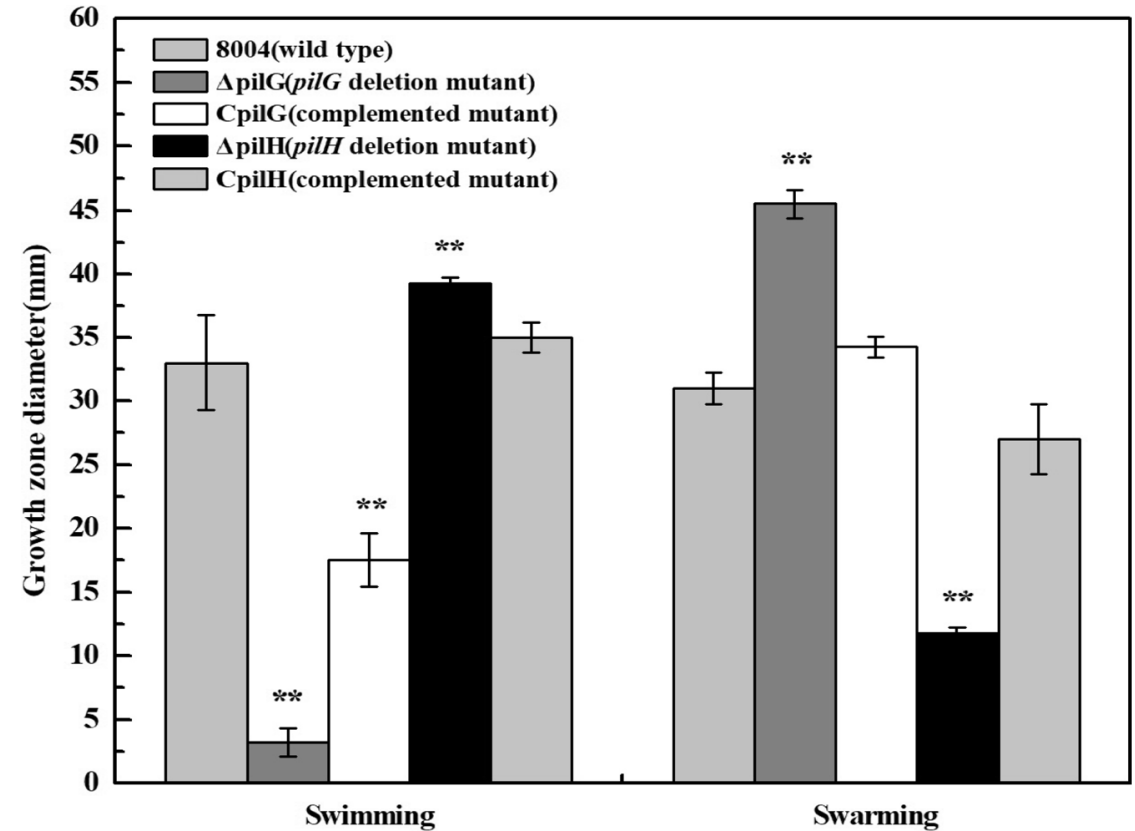

Fig. 1 PilG and PilH antagonistically regulate swimming and swarming motility in Xcc. a Examination of swimming and swarming motility. i and ii Examination of swarming motility for mutation in genes pilG and pilH. Strains were inoculated onto 'swarm' plate (NY medium containing $2 \%$ glucose and $0.6 \%$ agar) then incubated at $28{ }^{\circ} \mathrm{C}$ for 3 days. iii and iv Examination of swimming motility for mutation in genes pilG and pilH. Strains were stabbed into 'swim' plate $\left(0.03 \%\right.$ Bacto peptone, $0.03 \%$ yeast extract and $0.28 \%$ agar) then incubated at $28{ }^{\circ} \mathrm{C}$ for 3 days. $\mathbf{b}$ The diameter of the colony 8004, $\Delta$ pilG, CpilG, $\Delta$ pilH and CpilH on swimming and swarming plates. Significance was tested by Student's t test ${ }^{*}$ and ${ }^{* *}$ represent significance at $P<0.05$ and 0.01 , respectively) 
PilG and PilH influence chemotaxis and virulence in Xcc To explore if PilG and PilH manipulate additional specific functions that are known to be associated with motility and virulence, we conducted a series of phenotypic tests to assess growth, EPS, chemotaxis and virulence in Xcc (see Methods).

As shown in Additional file 2: Figure S2, production of EPS in $\Delta$ pilG was similarity to the wild type but $\Delta$ pilH decreased production of EPS greatly, and then no influence on growth between mutants and wild type in NY medium. Additionally, differences were seen when the wild-type strain, the $\Delta$ pilG and $\Delta$ pilH mutants and the complemented strains ability to sense and respond chemotactic agents was assessed. For these experiments, a simplified capillary assay for the qualitative analysis of chemotaxis was used (see Methods). Here eight selected chemoattractant (two inorganic salts $\left[\mathrm{CaCl}_{2}, \mathrm{MgCl}_{2}\right]$, three carbohydrates [maltose, glucose, sucrose] and two amino acid [serine, arginine]) and a negative control $\left(\mathrm{H}_{2} \mathrm{O}\right)$ were used to assess the chemotactic response of $X c c$. The results revealed that $\Delta$ pilG and $\Delta$ pilH strains exhibited a significantly lower response to $\mathrm{MgCl}_{2}$ and sucrose compared with wild-type (Fig. 2a). However, no differences between complementary strains CpilG and $\mathrm{CpilH}$ and wild-type were seen. These results indicated that PilG and PilH have no effect on growth in NY medium and regulate the chemotactic response of Xcc.

Interestingly, alterations were seen when strains were assessed for their virulence. The results showed that the $\Delta$ pilG and $\Delta$ pilH mutants displayed decreased diseased leaves rate via spraying inoculation onto radish leaves (Fig. 2b), but the virulence was no differences for $\Delta$ pilG and $\Delta$ pilH mutants though leaf-clipping to infect radish leaves (data not shown). To determine if growth in planta contributes to plant pathogenicity, we determined the growth of strains. As shown in Fig. 2c, $\Delta$ pilG and $\Delta$ pill have slower growth in planta compared to wildtype and the corresponding complemented strains. These data indicated that PilG and PilH have effects on pathogenicity mainly to be colonized and grown on host tissues.

Taken together, these findings suggested that PilG and $\mathrm{PilH}$ contribute to the regulation of chemotaxis and virulence in $X c c$ under the conditions tested. Despite these observations, the mechanism of regulation of chemotaxis and virulence by $\mathrm{PilG}$ and $\mathrm{PilH}$ remains to be further understood.

PilG and PilH have an influence over the expression of genes involved in chemotaxis, flagellar biosynthesis and pilus assembly in Xcc

The findings outlined above showed that PilG and PilH play complex roles in the regulation of pilus-dependent and flagellum-dependent motility and other associated phenotypes. To gain a greater understanding of the regulatory role of PilG and PilH in Xcc a set of global gene expression profiles were generated using RNA-seq and RT-PCR. For these experiments, total RNA was purified from the wild-type, $\Delta$ pilG and $\Delta$ pilH strains grown to the mid-exponential phase $\left(\mathrm{OD}_{600}=1.0\right)$ in NYG medium, and tested quantity and quality (see Methods).

Following bacterial RNA extraction and sequencing, differential gene expression analysis was conducted on the generated data (see Methods). A false discovery rate of FDR $\leq 0.05$, and $\left|\log _{2} \mathrm{FC}\right|\left(\log _{2}\right.$ of the fold changes $) \geq 1$ was considered for differentially expressed genes. Comparison of transcriptome data between the wild-type, $\Delta$ pilG and $\Delta$ pilH strains reveal significant differences. In the absence of PilG a total of 152 genes were altered in which 125 genes were down-regulated and 27 genes were up-regulated (Additional file 7: Table S2). However, in the absence of PilH a total of 195 genes revealed significantly altered expression in which 106 were downregulated and 89 were up-regulated genes (Additional file 8: Table S3). Interestingly, only 60 expressed genes appear to co-regulated by PilG and PilH (Fig. 3a). To validate the transcriptome data and confirm the changes in the coregulated genes a total of 18 genes were selected and the expression changes were validated by semi-quantitative RT-PCR (Additional file 3: Figure S3). These results confirmed that the differential gene expression analyses based on our transcriptome are reliable.

The genes that showed expression changes were subjected to functional categorization using KEGG using the Xcc 8004 genome annotation [20]. Based on biological functions analysis, all of the genes that changed were mainly catalogued into 13 functional categories that included signal transduction, biosynthesis and metabolism (Fig. 3b). Despite genes being ascribed to a broad number of functional categories majority of regulated genes were predicted to encode hypothetical proteins or did not have a functional category assigned to date. This included 85 of 152 genes regulated by PilG and 84 of 195 genes regulated by PilH. While 29 of the 60 genes co-regulated by both PilG and PilH were predicted to encode hypothetical proteins (Fig. 3c).

Interestingly, examination of the remaining co-regulated genes showed they were associated with functions relating to motility, specifically chemotaxis, flagellum and pilus assembly and function (Additional file 9: Table S4). Consistent with the motility phenotypes attributed to be under the control of PilG and PilH it was clear that these genes were divergently regulated (Fig. 3c). For example, 16 chemotaxis-associated genes $\left(X C_{-} 2320, X C_{-} 2302, X C_{-}\right.$ 2311, XC_2309, XC_2223, XC_1413, XC_0638, XC_2321, $X C \_2306, X C \_2318, X C_{-} 1410, X C_{-} 2303, X C_{-} 2314, X C_{-}$ 1414, XC_2313, XC_2298), and 4 flagellum-related genes 


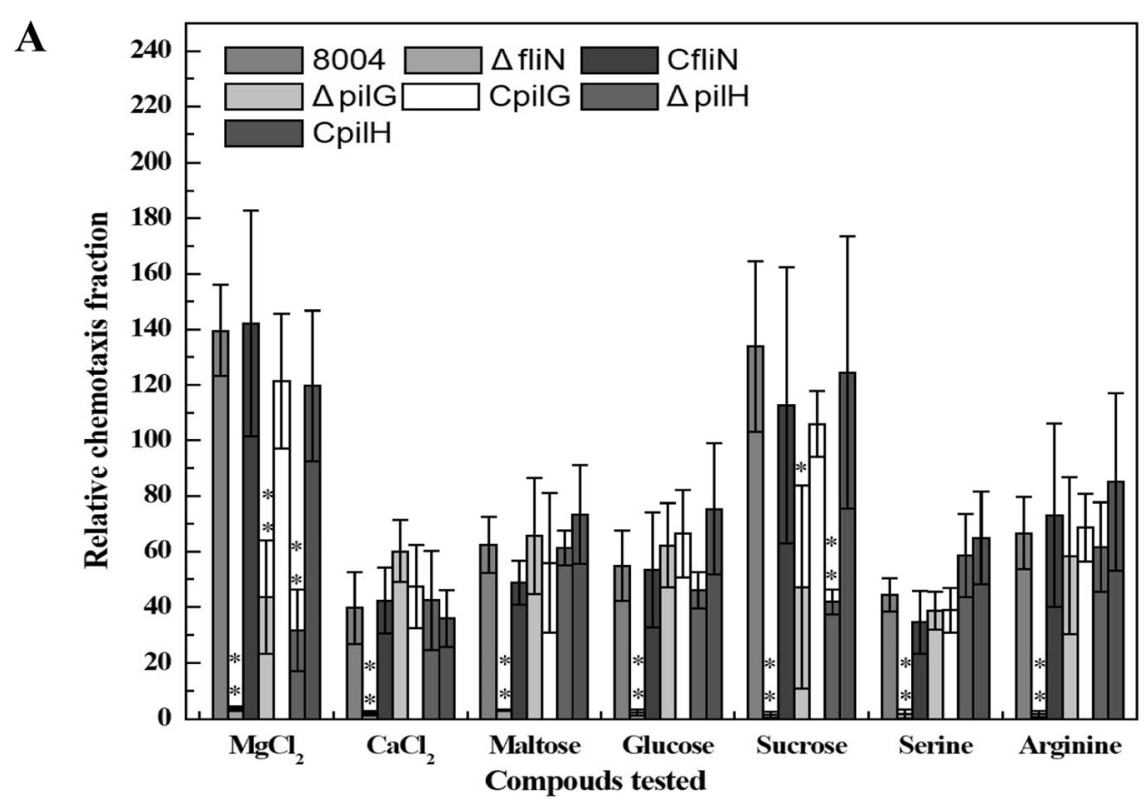

B

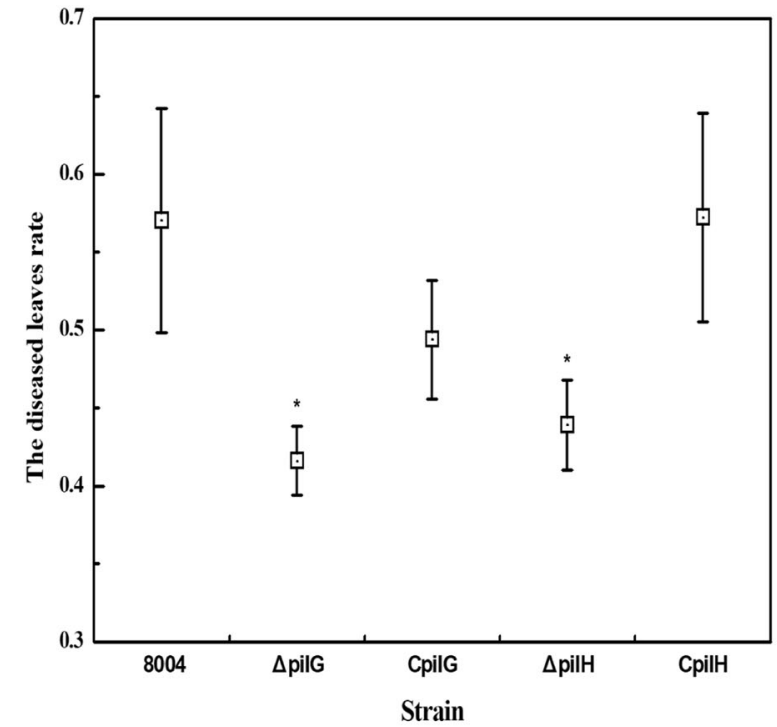

C

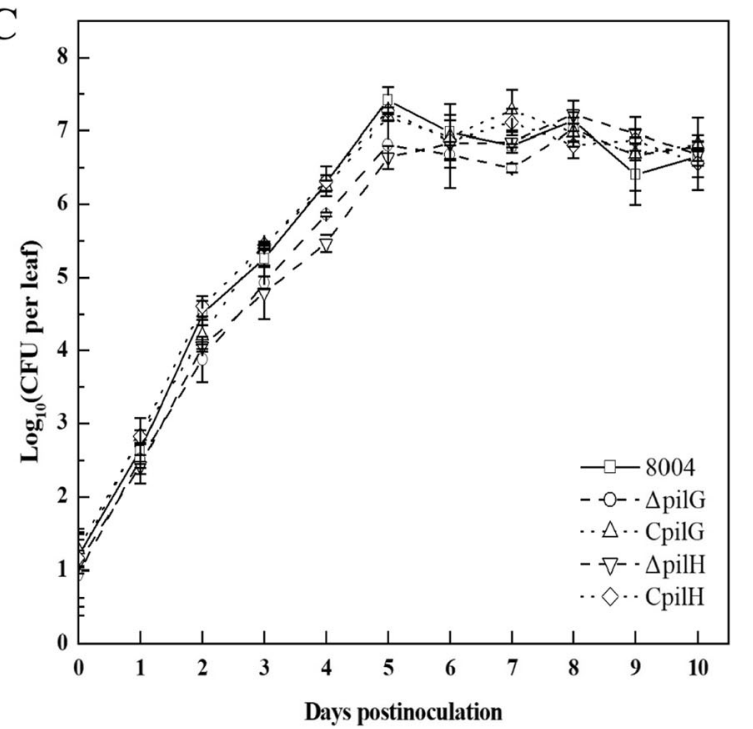

Fig. 2 PilG and PilH influence diverse cellular processes including chemotaxis and virulence. a Chemotaxis response assay. The strains were diluted and performed simplified capillary assays for 8 chemoattractant and counted bacterial CFUs then incubating at $28^{\circ} \mathrm{C}$ for 3 days. Relative chemotaxis fraction was calculated CFU in test capillary vs CFU in control buffer capillary. $\mathbf{b}$ The virulence of Xcc strains. The strains were diluted and sprayed onto leaves of 100 radishes showing similar growth, and counted diseased leaves rate after 10 days. Additionally, each strain was repeated three times. $\mathbf{c}$ In-plant growth curve. The strains were diluted and inoculated to Chinese radish by leaf-clipping. At intervals of 1 day, four clipped leaves for every group of inoculated plants were collected and homogenized, and then diluted to plate on NYG plates. The colonies were counted after 3 days of incubation at $28^{\circ} \mathrm{C}$. Significance was tested by Student's t test (* and ** represent significance at $P<0.05$ and 0.01 , respectively)

(XC_2245, XC_2264, XC_2231, XC_2246) were found to be down-regulated in the $\triangle$ pilG mutant, but up-regulated in the $\triangle$ pilH mutant (Additional file 9: Table S4). Furthermore, pilI (XC_1185) and pilJ (XC_1186) involved in pilus-dependent motility were down-regulated in the $\Delta$ pilH strain but were unchanged in the $\Delta$ pilG strain.
Taken this data together it is clear the impact of PilG and PilH on the expression of specific genes at the transcriptional level accounts for the phenotypes seen in the $\Delta$ pilG and $\Delta$ pilH strains. Furthermore, these results indicate PilG and PilH control pilusdependent and flagellum-based motility by potentially 


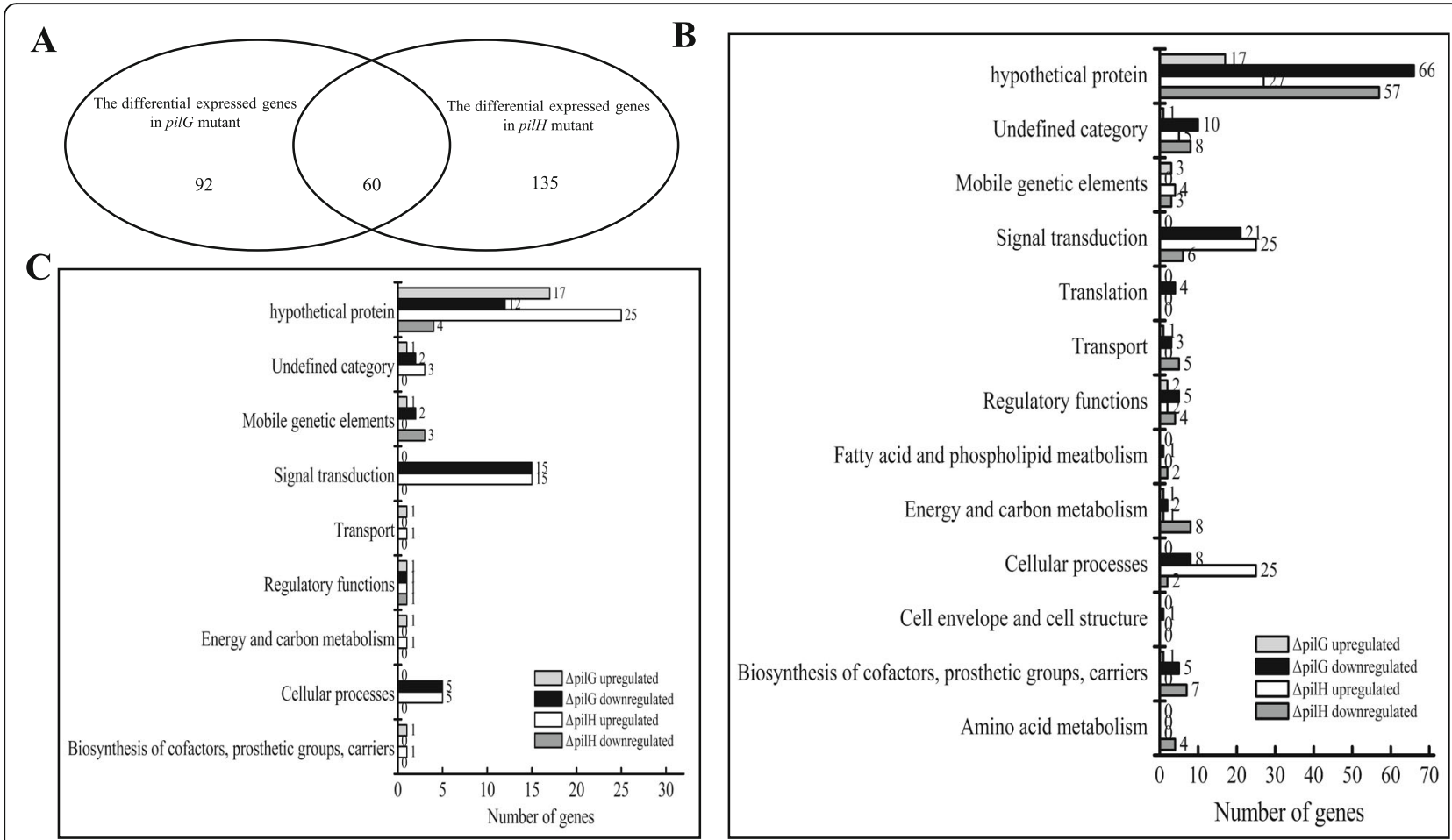

Fig. 3 Functional categories of the differential expressed genes in two mutant backgrounds. a The venn diagram showed the overlap between the differential expressed genes in two mutant backgrounds. $\mathbf{b}$ Functional categories of the differential expressed genes in two mutant backgrounds. The differential expressed genes from Additional file 7: Table S2 and Additional file 8: Table S3 were broadly categorized according to their biological function. c Functional categories of overlap of the differential expressed genes in two mutant backgrounds. The differential expressed genes from Additional file 9: Table \$4 were broadly categorized according to their biological function. Each bar represents the number of differential expressed genes in each category of Xcc 8004 genome

opposingly regulating genes involved in flagellar biosynthesis and pilus assembly.

\section{Mutation in genes that encoding structural elements of the pili, flagellum and chemotaxis systems reveal similar phenotypes to pilG and pilH in Xcc}

The gene clusters encoding proteins needed to generate the flagellar and pili systems in Xcc have previously been examined in strains Xc17 and ATCC 33913 [19]. In the strain used in the current study was examined for the presence of flagellar and pili genes along with known regulatory genes. By these analyses, we found the presence of one cluster of motility-related (flagellar and chemotaxis) genes, and several pili-related genes. In order to examine the impact of these genes on the phenotypes that appear to be controlled by PilG and PilH, the effects of mutation of specific genes were assessed (see Methods). Specifically, we constructed in-frame deletion mutations in flagellum-assembled genes fliM and fliN (XC_2267 and XC_2268), a pili-assembled gene pilB $\left(X C_{-} 1060\right)$ and chemotaxis-associated genes $\left(X C_{-} 1414\right.$, $X C_{-} 2302$ and $X C_{-}$2306) (see Methods). These strains were designated $\Delta$ fliM, $\Delta$ fliN, $\Delta$ pilB, $\Delta$ cheA, $\Delta$ cheY and $\Delta 2306$, respectively (Additional file 6: Table S1). In tandem, these mutants were complemented and the resulting strains were named CfliM, CfliN, CpilB, CcheA, CcheY and C2306 (Additional file 6: Table S1). This functional genomic examination of those structural elements of the pili, flagellum and chemotaxis systems that may indirectly contribute to phenotypes of pilG and pilH mutants.

The pilus-dependent and flagellum-based motility of each mutant were tested (see Methods). Mutation of fliM (XC_2267), fliN (XC_2268) and cheY (XC_2302) led $80 \%$ loss of flagellum-based swimming compared to the wild-type (Fig. 4a and b) and their corresponding complementary strains could be back to $60 \%$ of the wild type in phenotype. Interestingly, mutation of pilB led to an increase of $20 \%$ approximately in swarming compared to the wild-type (Fig. $4 \mathrm{a}$ and b), and then complementation restored the phenotypes towards wild-type (Fig. 4a and b). None of the other mutants showed any significant difference in motility compared to wild-type.

In addition, alterations were seen when strains were assessed for their response to chemotactic agents (see Methods). The two chemotaxis-associated genes $\left(X C_{-}\right.$ 1414 and $X C_{2} 2306$ ) were found to exhibit a significant difference in chemotaxis response. Analysis showed that 


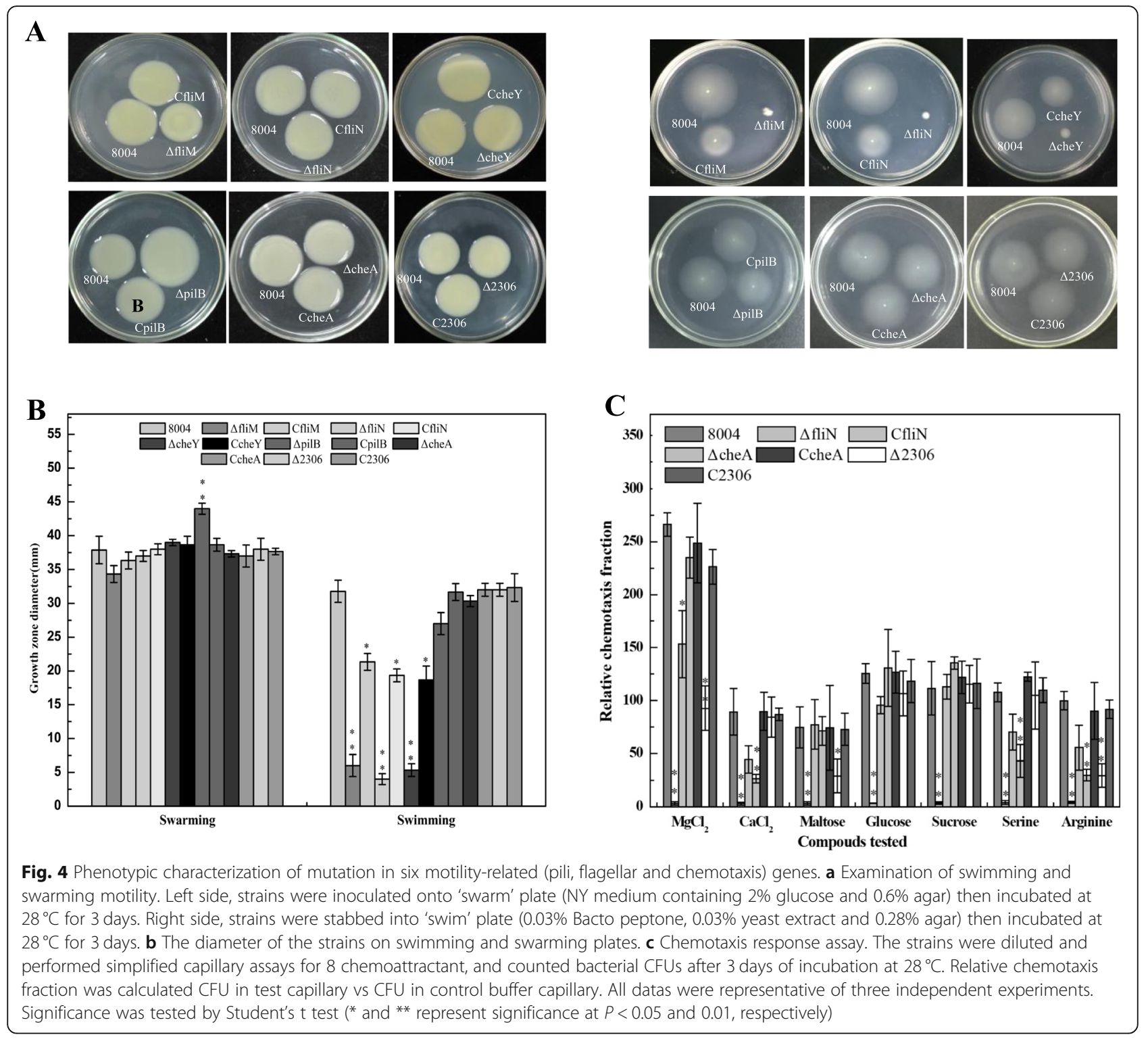

there were significant differences in $\Delta$ cheA $\left(X C_{-} 1414\right)$ compared to wild-type toward $\mathrm{CaCl}_{2}$, serine and arginine (Fig. 4c). While the $\Delta 2306$ (XC_2306) showed a difference in movement toward $\mathrm{MgCl}_{2}$, maltose and arginine compared to wild-type (Fig. 4c).

PilG and PilH interact directly with district subsets of proteins that potentially account for their regulatory role The majolity of CheY-like proteins (contain a stand-alone REC domain) have been shown to regulate motility by intermolecular interactions with motor proteins. While other stand-alone REC domains, such as Bacillus subtilis Spo0F, have been shown to function as phosphorylated intermediate in phosphorelay pathways [21-24]. The observations discussed above indicate that PilG and PilH regulate the expression of genes that indirectly influence
Xcc motility but also that a set of structural elements of the pili, flagellum and chemotaxis systems that may contribute to phenotypes of pilG and pilH mutants.

To examine if PilG and PilH potentially interact specifically with proteins, we initially employed coimmunoprecipitation (co-IP) coupled with and mass spectroscopy (see Methods). For these experiments a $3 \times$ Flag-tagged fusion protein $3 \times$ Flag::PilG (or PilH) was constructed (Additional file 6: Table S1), where a $3 \times$ Flag-tag was fused to the 5 'end of the pilG gene (or pilH gene) and cloned into the vector pLAFR3. These constructs were introduced into strains of interest including the respective mutant ( $\Delta$ pilG or $\Delta \mathrm{pilH})$, and the complemented strain (CpilG or $\mathrm{CpilH}$ ) for not expressing $3 \times$ Flag-tagged fusion proteins as a negative control (Additional file 6: Table S1). A western blot assay 
confirmed that the $3 \times$ Flag::PilG (or PilH) fusion protein complex could be eluted from strains for expressing $3 \times$ Flag-tagged fusion protein, but not the control strain CpilG (or $\mathrm{CpilH}$ ) for not expressing $3 \times$ Flag-tagged fusion proteins (Fig. 5a). The MScoupled co-IP experiment was repeated twice and take the same results but removing results from negative control as the candidate target proteins. The $3 \times$ Flag::PilG (or PilH) fusion protein complexes purified from the $\Delta$ pilG mutant the identities of 6 interacting proteins could be established by mass spectrometry (Fig. 5b). Interestingly, the $3 \times$ Flag::PilH fusion identified a completely different set of 7 interacting proteins (Fig. 5b). These proteins were shown

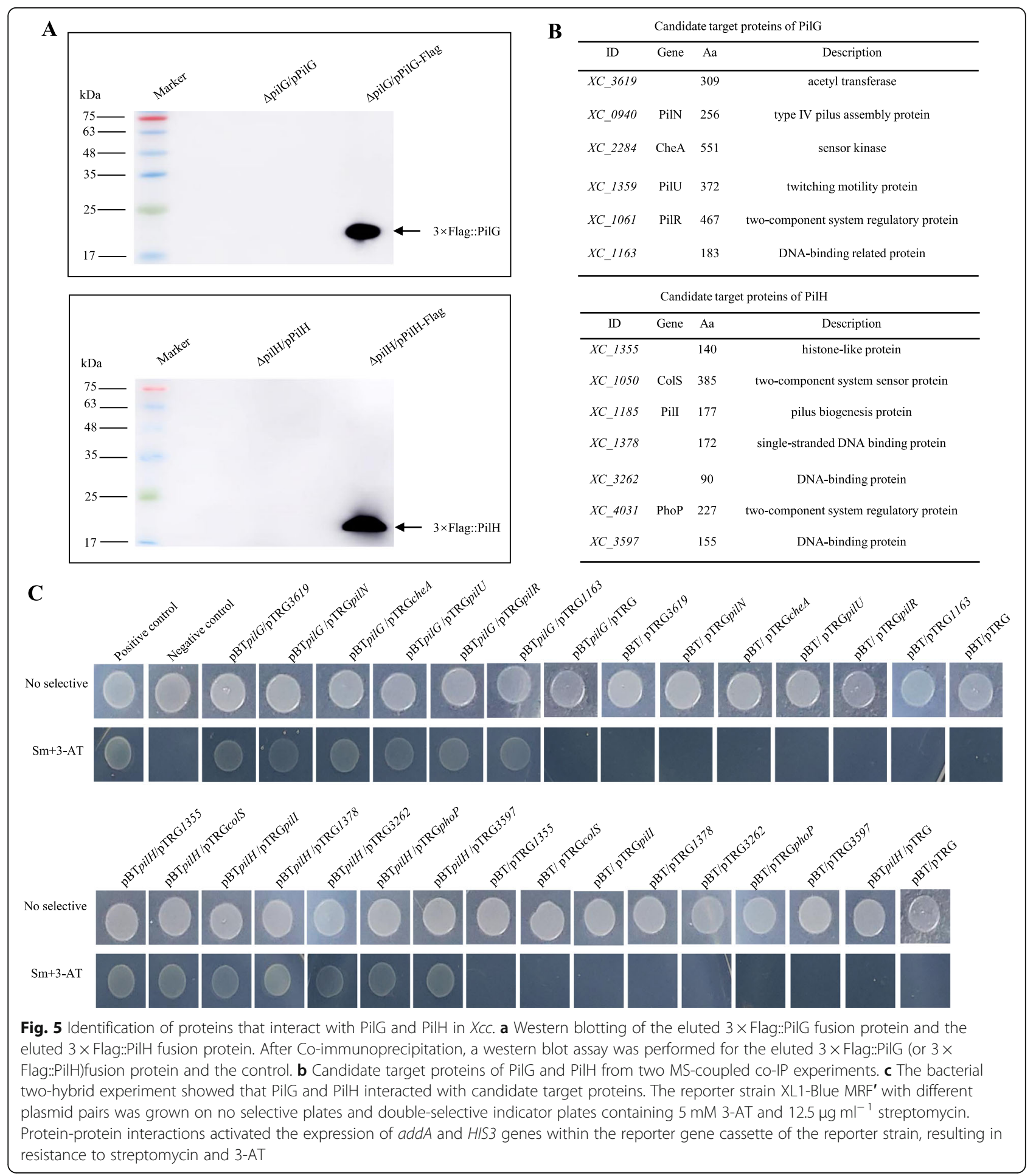


to have roles in signalling (sensor kinases), regulation (DNA-binding regulators) and importantly motility.

The co-IP analysis was extended and validated by using a bacterial two hybrid and biotinylated proteinprotein blotting experiments. Importantly, the PilG and $\mathrm{PilH}$ protein were shown to interact with their respective proteins using bacterial two hybrid assay (Fig. 5c). PilG confirmed directly interaction with XC_0940, XC_1061, XC_1163, XC_1359, XC_2284 and XC_3619. When PilH was tested it showed interactions with XC_1050, XC_ 3262, XC_1185, XC_1355, XC_1378, XC_3597 and XC_ 4031. Interestingly, both $\mathrm{PilG}$ and $\mathrm{PilH}$ proteins interacted with FilN by using bacterial two hybrid and biotinylated protein-protein blotting experiments (Additional file 4: Figure S4). All interactions were detected for a second time. Taken together, these data suggested that PilG and PilH interact with two distinct subsets of proteins that have roles in signalling, regulation and importantly motility.

\section{Functional genomic assessment of genes encoding protein that interact with PilG and PilH identifies a novel regulator involved in Xcc motility}

As mutation of pilG and pilH lead to an alteration in motility and associated traits in $X c c$, it follows that mutation of genes that encode proteins that interact with these regulators may have a role in motility. This was confirmed by our previous work which has shown that mutation of genes encoding PilN (XC_0940), PilU (XC_ 1359), PilR (XC_1061), XC_3262 and PhoP (XC_4031) have been shown to play a role Xcc motility $[17,25-27]$.

In order to examine this the other proteins that interacted with PilH and PilG, the effects of mutation on selected genes were assessed swimming and swarming motility (see Methods). The strain carrying a deletion in $X C_{-} 1185$ demonstrated the most profound effect on swarming motility (Additional file 5: Figure S5). $X C_{-}$ 1185 encodes a signalling protein containing a CheW domain and homologs of this protein are named Pill in strains of Pseudomonas sp. Importantly, swarming motility was restored towards wild-type by complementation in trans with a wild-type copy (Additional file 5: Figure S5).

Taken together, these findings suggested that proteins that interact with PilG and PilH may regulate motility in a similar indirection under the conditions tested. Furthermore, a new factor Pill (XC_1185) which interacts with PilH directs has partly the same phenotypes. Interestingly, this protein has homologues in other plant associated bacteria, including other Xanthomonas species. However, despite these observations, the mechanism of regulation by PilG and PilH in these cases remains enigmatic and the question of how PilG, and in particular $\mathrm{PilH}$, influences these proteins regulatory roles is yet to be understood.

\section{Discussion}

The surface appendages flagellum and pili are the most commonly studied motility devices in bacteria. Polar flagella are known to act as 'helical propellers', whereas the pili act as 'grapnel' or 'anchors' [28]. These systems can also contribute to biofilm formation, surface attachment and chemotaxis. In many bacteria, PilG and PilH have been shown to regulate pilus-dependent motility [14, 15, 29]. In the current study, we present evidence demonstrating the roles of PilG and PilH in the regulation of motility of Xcc are more complex than previously described in other Gram-negative bacteria. The data consistent with the hypothesis that PilG and PilH have antagonistic regulatory effects on flagellum-dependent and pili-dependent motility, where PilG and PilH appear to exert their regulatory influence by interacting directly with a subset of proteins involved in flagellar biosynthesis and pilus assembly or controlling gene expression.

Through the construction and complementation of deletion mutants, we revealed that PilG positively regulates flagellum-dependent motility while and negatively regulating pili-dependent motility. Conversely, PilH was shown to negatively regulate flagellum-dependent behaviour while and positively regulating pili-dependent motility. In addition to motility, little is known about specific functions of single-domain response regulators [30], so we were looking for functional information of PilG and PilH though transcriptional regulation and protein-protein interaction mediated regulation. These observations were supported by transcriptome analyses that showed PilG up-regulating many genes involved chemotaxis and flagellar biosynthesis but these genes were inversely regulated by PilH. It is clear that PilG and $\mathrm{PilH}$ appear to play unique roles in controlling motility in Xcc. A better understanding of how PilG and PilH might influence motility was revealed by the MScoupled co-IP experiments. We found that PilG could interact with PilN, PilR, PilU, CheA, XC_3619 and XC_ 1163. While PilH interacted with Pill, ColS, PhoP, XC_ 1355, XC_1378, XC_3262 and XC_3597. Importantly, our previous work has shown that mutation of genes encoding PilN (XC_0940), PilU (XC_1359), PilR (XC_ 1061), XC_3262 and PhoP (XC_4031) have been shown to play a role $X c c$ motility [17, 25-27]. Furthermore, additional mutagenesis revealed that a new factor Pill (XC_1185) which interacts with PilH has partly the same phenotypes in $X c c$.

It has been demonstrated in $P$. aeruginosa that $\mathrm{PilH}$ directly influences Pill and PilJ which in turn controls the production of PilA [15]. Therefore, this might be one reason why $\mathrm{PilH}$ in $\mathrm{Xcc}$ may regulate pili-dependent motility as it interacts with Pill. Nonetheless, the way PilG independently regulates pili-dependent motility is not clear. The three pili related proteins that PilG 
interacts with are PilN, a pili-assembly protein, which may form an inner membrane subcomplex with PilO and PilP to influence alignment of the secretin for the pilus assembly machinery [31]; PilR, shown to function as a transcriptional activator of biosynthesis of PilA in Xanthomonas axonopodis pv. citri [32] and PilU, a ATPase able to provide energy for extension or contraction of pili $[26,33]$. However, the RNA-Seq data revealed that PilG did not affect the expression of PilA, indicating that PilG might regulate swarming via interacting with PilN and PilU to affect assembly. Further work is required to confirm this.

Although PilG and PilH appear to interact directly with subsets of proteins, the influence on these proteins is still very much in question. Many proteins containing stand-alone REC domains, such as B. subtilis Spo0F, have been shown to function as phosphorylated intermediate in phosphorelay pathways [21-24]. Maybe PilG and PilH accept phosphoryl groups from the sensor kinase CheA and ColS, respectively. However, PilG and $\mathrm{PilH}$ accepting phosphoryl groups from CheA and ColS provides clues to how this protein is involved in regulation of flagellar motility. It has been demonstrated in E.coli that the direction of flagellar rotation is regulated via phosphorylated CheY bound to the N-terminal segment of FliM [21-24], and that the C-terminal segment of FliM is flexible enough to allow subsequent binding to a site on FliN in the vicinity of the hydrophobic patch [34], finally it destabilizes the interaction between FliM and the C-terminus of FliG and FliG is bound to MotA that causes the flagellar rotation $[35,36]$. However, a more recent work has found that chemotaxis signalling protein $\mathrm{CheY}$ binds to the rotor protein FliN to control the direction of flagellar rotation in E coli [37]. Here a direct interaction between PilG and flagellar proteins FliN was detected based on bacterial two-hybrid assay and pull-down biotinylated protein-protein assay, suggesting that PilG may influence flagellum-dependent motility though interaction with FliN. However, the role of PilH in flagellum-dependent motility remains enigmatic. Work in P. aeruginosa has suggested that PilH may not directly regulate flagellum-based motility but act as a phosphate sink [38]. This remains to be confirmed in Xcc.

In addition to the influence over flagellum-dependent and pili-dependent motility, pilG and pilH deletion mutants showed decreased virulence and chemotaxis ability for $\mathrm{MgCl}_{2}$ and sucrose, suggesting that these signalling protein interfaces with multiple signal transduction circuits in Xcc. Transcriptome analysis revealed that the chemotaxis-associated genes were downregulated in pilG mutant and upregulated in pilH mutant, indicating that pilG and pilH may promote chemotaxis ability of $X c c$ though altering expression of chemotaxis-associated genes. Despite these observations, the mechanism of regulation of virulence and chemotaxis by PilG and PilH remains to be further understood.

In summary, our study has identified that PilG and $\mathrm{PilH}$ as two multifunctional regulators that control diverse cellular processes including swarming, swimming, surface adherence and chemotaxis in Xcc. Specifically, the influence of PilG and PilH on motility in $\mathrm{Xcc}$ is likely the result of two fold where: (1) PilG and PilH antagonistically affect the expression of genes involved in flagellum and pili-dependent motility; (2) PilG and PilH interact with subsets of proteins that are involved in pilidependent motility. Despite these advances further studies are needed to examine the role of PilG and PilH in the regulation of the additional phenotypes they have been shown to regulate but also suggest several other questions in to be addressed: What are the environmental cues that activate the expression and activity of PilG and PilH? Does PilG and PilH regulate differently during plant colonization? How does PilG and PilH directly influence the proetins they interact with? How does PilG and PilH affect gene expression in? Is PilG and PilH involved in phosphotransfer? Do PilG and PilH have conserved protein interaction sites?

\section{Conclusions}

In this work, we found that mutation of the gene encoding PilG and PilH antagonistically control flagellumdependent and pili-dependent motility, and present evidence showing that PilG and PilH are involved in other cellular processes. In summary, we demonstrate that (1) PilG and PilH antagonistically affect the expression of genes involved in flagellum and pili-dependent motility; (2) PilG and PilH interact with subsets of proteins that are involved in pili-dependent motility.

\section{Methods}

\section{Bacterial strains and culture conditions}

The bacterial strains and plasmids used in this study were listed in Additional file 6: Table S1. Escherichia coli strains were routinely grown in Luria-Bertani broth at $37^{\circ} \mathrm{C}$. Xcc strains were grown at $28^{\circ} \mathrm{C}$ in NYG medium (peptone, $5 \mathrm{gL}^{-1}$; yeast extract, $3 \mathrm{gL}^{-1}$; and glycerol, $20 \mathrm{gL}^{-1}, \mathrm{pH}$ 7.0) [39]. Antibiotics were added at the following concentrations as required: kanamycin (Kan) $25 \mu \mathrm{gml}^{-1}$, rifampicin (Rif) $50 \mu \mathrm{gml}^{-1}$ and tetracycline (Tet) $5 \mu \mathrm{gml}^{-1}$ for Xanthomonas spp.

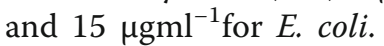

\section{Construction of in-frame deletion mutants and its genomic integrated complemented strains}

In-frame deletion mutant was constructed by two exchange steps using the plasmid pK18mobsacB [40]. For construction of Xcc pilG deletion mutant, 521-bp upstream and 
327-bp downstream fragments flanking pilG (XC_1183) were amplified using the total DNA of the $X c c$ wild type strain 8004, respectively. Primers were modified to give EcoRI-, XbaI- or HindIII-compatible ends (underlined) (Additional file 10: Table S5). Two fragments were cloned together into the vector $\mathrm{pK} 18 \mathrm{mobsacB}$, and then the recombinant plasmid was introduced into the Xcc strain 8004 by triparental conjugation. The trans-conjugants were screened for selective agar plates containing $5 \%$ sucrose. The obtained pilG deletion mutant was further confirmed by PCR and named $\triangle$ pilG. Other deletion mutants were constructed in the same way as mutant $\Delta$ pilG.

For construction of complemented mutant, a DNA fragment containing the encoding region of pilG was amplified by PCR using the primers CGF/R, and then the amplified DNA fragment was cloned into the plasmid pLAFR3 (Additional file 6: Table S1) to generate recombinant plasmid. While the recombinant plasmid was introduced into the mutant $\Delta$ pilG by triparental conjugation, generating complemented strain CpilG. Other complemented strains were constructed in the same way as the complemented strain CpilG.

\section{Motility assay}

For examination of swimming motility, strains were stabbed into $0.28 \%$ agar plate containing $0.03 \%$ Bacto peptone and $0.03 \%$ yeast extract using toothpick then incubated at $28{ }^{\circ} \mathrm{C}$ for 3 days. To analyse swarming motility, strains grown overnight in NYGB medium $\left(\mathrm{OD}_{600}=\right.$ 1.0) were inoculated onto NY plate containing $2 \%$ glucose and $0.6 \%$ agar then incubated at $28^{\circ} \mathrm{C}$ for 3 days.

\section{Chemotaxis response assay}

To analyse chemotaxis response of $X c c$, we used a simplified capillary assay [41]. The strains were grown in the NYG medium for overnight and diluted to $\mathrm{OD}_{600}$ of 1 , and then $100 \mu \mathrm{l}$ diluted culture was sucked into a disposable pipette tip and the chemotaxis capillary containing the chemoattractant was attached to bacterial suspension in disposable pipette tip steadily at $28^{\circ} \mathrm{C}$. Two hours later, the chemoattractant in the chemotaxis capillary was blown out and diluted to 0.0001, and $100 \mu \mathrm{l}$ diluted culture was plated onto NYGA plate. Bacterial CFUs were counted after incubating at $28^{\circ} \mathrm{C}$ for 3 days. $\mathrm{H}_{2} \mathrm{O}$ was used as a control buffer for test capillary. Relative chemotaxis fraction was calculated CFU in test capillary vs CFU in control buffer capillary.

\section{Plant inoculation assay}

The virulence of Xcc to Chinese radish (Raphanus sati$v u s)$ was tested by the leaf-clipping or leaf-spraying method. The strains were grown in the NYG medium for overnight and diluted to $\mathrm{OD}_{600}$ of 0.001 , and then inoculated to Chinese radish by leaf-clipping or leaf- spraying method. Lesion length was measured or diseased leaves rate was counted for 10 days later.

\section{Growth curve of Xcc strains}

For in-NY growth curve, the strains were inoculated into NYG medium with the same final density of 0.01 , growth of the strains was diluted and plated on NYG plates at intervals of $4 \mathrm{~h}$. The living cells were counted after 3 days of incubation at $28^{\circ} \mathrm{C}$.

Bacterial in planta growth was tested as previously described [42]. The strains were grown in the NYG medium for overnight and diluted to $\mathrm{OD}_{600}$ of 0.001 , and then inoculated to Chinese radish by leaf-clipping. At intervals of 1 day, four clipped leaves for every group of inoculated plants were collected and homogenized, and then diluted NYG medium to plate on NYG plates. The colonies were counted after 3 days of incubation at $28^{\circ} \mathrm{C}$.

\section{EPS assays}

To estimate EPS production, the strains were inoculated into $100 \mathrm{~mL}$ NY liquid medium containing glucose $(2 \% \mathrm{w} /$ v) at $28^{\circ} \mathrm{C}, 200 \mathrm{rpm}$ for 5 days. EPS was precipitated from the culture supernatant with ethanol and dried at $55^{\circ} \mathrm{C}$ and weighed as described [5]. For analysis of EPS production on plates, the strains were grown in the NYG medium for overnight and diluted to $\mathrm{OD}_{600}$ of 1 , and then $3 \mu$ diluted culture was inoculated onto the NY plates containing $2 \%$ glucose and $2.0 \%$ agar. The results were observed after 5 days of incubation at $28^{\circ} \mathrm{C}$.

\section{Global transcriptional analysis}

Total RNA was sent to Majorbio-Shanghai with RNA isolated from cultures of $X c c$ strains grown to an $\mathrm{OD}_{600}$ of 1.0 in NYGB medium. and tested quantity and quality on a Nanodrop spectrophotometer ND-8000 and Agilent 2100 bioanalyzer respectively. The sample was subjected to RNA sequence in an Illumina HiSeq2000 platform at a company (Majorbio, Shanghai, China).

\section{Semi-quantitative PCR}

RNA was isolated from $X c c$ strains grown to $\mathrm{OD}_{600}$ of 1.0 in NYG medium using Omega's RNA kit according to manufacturer's protocol. The cDNA was synthesized from total RNA using Superscript III First Strand Synthesis kit (Invitrogen) according to manufacturer's protocol. Semi-quantitative PCR was performed with different primer sets using cDNA as templates, 16S rRNA of Xcc 8004 as an internal control. Finally, PCR products were separated by electrophoresis on a $1.2 \%$ agarose gel.

\section{Co-immunoprecipitation}

In first, plasmid for expressing $3 \times$ Flag-tagged fusion protein $(3 \times$ Flag::PilG) was constructed, which was fused with the coding region of $3 \times$ Flag-tag at the $5^{\prime}$ end of 
the pilG gene and cloned into pLAFR3, and then the fused plasmid were introduced into $\Delta$ pilG by triparental conjugation. The Flag-tagged fusion protein produced by the fused plasmids in $\Delta$ pilG, and complemented strain CpilG for not expressing $3 \times$ Flag-tagged fusion protein as a negative control.

The strains were grown in the NYG medium for overnight and collected by centrifugation at $4{ }^{\circ} \mathrm{C} 4000 \mathrm{rpm}$ for $10 \mathrm{~min}$, and then washed with PBS buffer. Lysed the cells by resuspending them in $1 \mathrm{ml}$ of ice-cold lysis buffer $(25 \mathrm{mM}$ Tris- $\mathrm{HCl}$ pH 7.4, $100 \mathrm{mM} \mathrm{NaCl}, 1 \mathrm{mM}$ EDTA, 1\% NP-40, 10\% glycerine) containing protease inhibitor and incubated the resuspended cells on ice $2 \mathrm{~h}$. While centrifuged $10 \mathrm{~min}$ at $4{ }^{\circ} \mathrm{C}$, and transferred the supernatant into fresh tube. For each sample, it was added $50 \mu \mathrm{l}$ of ANTI-FLAG (agarose conjugated) and incubated in gently shaking at $4^{\circ} \mathrm{C}$ for $3 \mathrm{~h}$. Finally washed the agarose six times with ice-cold TBS buffer (or lysis buffer) and eluted the protein complexes by $0.25 \mathrm{M}$ glycine ( $\mathrm{pH} 2.5)$. Ultimately, we used a western blot assay to confirm $3 \times$ Flag-tagged fusion protein and then placed protein complexes to analyse by mass spectrometry facilities.

\section{Bacterial two-hybrid assay}

Protein-protein interaction was detected with the BacterioMatch II two-hybrid system (Stratagene, La Jolla, CA, USA). For example, the full length pilG, pilH, fliM and fliN was amplified by PCR using the total DNA of the Xcc wild-type strain 8004 as template and corresponding oligonucleotide set as primer (Additional file 10: Table S5), respectively. The pilG and pilH were cloned into the pTRG (prey), generating plasmids pTRGpilG and pTRGpilH. The fliM and fliN were cloned into the pBT (bait), generating plasmids pBTfliM and pBTfliN. To test protein-protein interaction, different combination of plasmids was co-transformed into the XL1-Blue $M R F^{\prime}$ reporter strain while and interaction was analysed by the manufacturer's instructions. The reporter strain XL1-Blue MRF' with different plasmid pairs was grown on no selective plates and double-selective indicator plates containing $5 \mathrm{mM} \mathrm{3-AT}$ and $12.5 \mathrm{\mu g} \mathrm{ml}^{-1}$ streptomycin. Protein-protein interaction activated the expression of $a d d A$ and HIS3 genes within the reporter gene cassette of the reporter strain, resulting in resistance to streptomycin and 3-AT.

\section{Pull-down assay}

Pull-down assay was performed by The ProFound pulldown biotinylated protein-protein interaction kit (Pierce, Rockford, IL, USA). The bait protein was biotinylated with sulfo-NHS-LC-biotin in PBS at room temperature for $40 \mathrm{~min}$, and then transferred to the centrifugal column and incubated with $20 \mu$ of streptavidin sepharose ${ }^{\text {tx }}$ beads for $40 \mathrm{~min}$ at $4{ }^{\circ} \mathrm{C}$. Beads bonded to bait protein were washed three times with TBS. The beads and $120 \mu \mathrm{g}$ prey protein were mixed and incubated $1 \mathrm{~h}$ for shaking gently at $4{ }^{\circ} \mathrm{C}$, prey protein was eluted and analysed by SDS-PAGE followed by Coomassie blue staining after washing beads three times with wash buffer.

\section{Supplementary information}

Supplementary information accompanies this paper at https://doi.org/10. 1186/s12866-020-1712-3

\begin{abstract}
Additional file 1: Figure S1. Identification of PilG and PilH in Xcc. (A) Sequence alignment of PilG in Xcc and other bacteria. The GenBank number of PilG homologue in Xanthomonas campestris pv. campestris is AAY48253; that in Pseudomonas aeruginosa is NP_249099; that in Lysobacter enzymogenes is BAV96685; that in Acinetobacter baumnnii is ABO13221. (B) Sequence alignment of PilH in XCC and other bacteria. The GenBank number of PilG homologue in Xanthomonas campestris pv. campestris is AAY48254; that in Pseudomonas aeruginosa is NP_249100; that in Lysobacter enzymogenes is BAV96686; that in Acinetobacter baumnnii is ABO13220. (C) Sequence alignment of PilG, PilH and CheY (ID: AAY49356) in Xanthomonas campestris pv. campestris with $\mathrm{CheY}_{\mathrm{EC}}$ (ID: 190906748) in E. coli.
\end{abstract}

Additional file 2: Figure S2. PilH positively regulates EPS production but PilG not, and they have no effect on the growth in NY medium. (A) Analysis of EPS production on plates. The strains were grown and diluted to $\mathrm{OD}_{600}$ of 1 , and then $3 \mu$ ldiluted culture was inoculated onto the NY plates containing $2 \%$ glucose and $2.0 \%$ agar. The results were observed after 5 days of incubation at $28^{\circ} \mathrm{C}$. (B) Production of EPS in Xcc strains. Mean weight of EPS extracted from the wild type strain, the pilG mutant, the pilH mutant and the corresponding complemented strains. (C) Growth curve of Xcc strains in NY medium. The strains were inoculated into NYG medium with the same final density of 0.01 , growth of the strains was diluted and plated on NYG plates at intervals of $4 \mathrm{~h}$. The living cells were counted after 3 days of incubation at $28^{\circ} \mathrm{C}$. Significance was tested by Student's t test ( ${ }^{*}$ and ${ }^{* *}$ represent significance at $P<0.05$ and 0.01 , respectively).

Additional file 3: Figure S3. Confirmation of RNA-Seq gene expression data by semi-quantitative RT-PCR. Note: Part of the differential expressed genes were performed to confirm the results of RNA-Seq by semi quantitative reverse-transcription PCR (semi RT-PCR). The expression levels of gene transcripts were calculated though the absolute value of $\log _{2}$ fold change $=1$ (equivalent to a fold change of 2 ). $\uparrow$ : up-regulated; $\downarrow$ : down-regulated.

Additional file 4: Figure S4. Bacterial two-hybrid experiment and pulldown assay demonstrated interaction between FliN and PilG or PilH. (A) Bacterial two-hybrid experiment showed that PilG and PilH interacted with FliN protein. The reporter strain XL1-Blue MRF' with different plasmid pairs was grown on no selective plates and double-selective indicator plates containing $5 \mathrm{mM} 3-\mathrm{AT}$ and $12.5 \mathrm{\mu g} \mathrm{ml}^{-1}$ streptomycin. Proteinprotein interactions activate the expression of addA and HIS3 genes within the reporter gene cassette of the reporter strain, resulting in resistance to streptomycin and 3-AT. (B) The pull-down assay demonstrated interaction between FliN and PilG or PilH in vitro. Lanes: 1, crude extract of BL21/pET30a after induction with IPTG; 2, crude extract of BL21/pET30a-PilG after induction with IPTG; 3, affinity-purified $\mathrm{His}_{6}$ - PilG protein; 4, crude extract of BL21/pET30a- $\mathrm{PilH}$; 5, affinity-purified $\mathrm{His}_{6}-\mathrm{PilH}$ protein; 6 , crude extract of M15/pQE30 after induction with IPTG; 7, crude extract of M15/pQE30- FliN after induction with IPTG; 8, affinity-purified

$\mathrm{His}_{6}$ - FliN protein; 9, pull-down of protein $\mathrm{His}_{6}$ - PilG by FliN; 10, pull-down of protein $\mathrm{His}_{6}-\mathrm{PilH}$ by FliN; 11, pull-down of protein $\mathrm{His}_{6}-\mathrm{HpaR} 1$ by FliN. $\mathrm{M}$, molecular mass marker.

Additional file 5: Figure S5. Mutation in gene pill influences swarming and swimming motility, but mutation in cols not. (A) Strains were stabbed into 'swim' plate $(0.03 \%$ Bacto peptone, $0.03 \%$ yeast extract and $0.28 \%$ agar) then incubated at $28^{\circ} \mathrm{C}$ for 3 days or inoculated onto 'swarm' 
plate (NY plate containing $2 \%$ glucose and $0.6 \%$ agar) then incubated at $28^{\circ} \mathrm{C}$ for 3 days. (B) The diameter of the colony $8004, \Delta$ pill, Cpill, $\Delta$ cols and Ccols on swimming and swarming plates. Significance was tested by Student's $\mathrm{t}$ test ${ }^{*}$ and ${ }^{* *}$ represent significance at $\mathrm{P}<0.05$ and 0.01 , respectively).

Additional file 6: Table S1. Bacterial strains and plasmids used in this work

Additional file 7: Table S2. The differential expressed genes of the pilG mutant strain $\triangle$ pilG in the rich medium NYGB.

Additional file 8: Table S3. The differential expressed genes of the pilH mutant strain $\triangle \mathrm{pilH}$ in the rich medium NYGB,

Additional file 9: Table S4. The overlap of differential expressed genes of the pilG mutant strain $\Delta$ pilG and the pilH mutant strain $\Delta$ pilH in the rich medium NYGB.

Additional file 10: Table S5. Primers used in this study.

\section{Abbreviations}

CFU: Colony forming unit; EPS: Extracellular polysaccharide; Kan: Kanamycin; Rif: Rifampicin; Tet: Tetracycline; Xcc: Xanthomonas campestris pv. campestris

\section{Acknowledgements}

Not applicable.

\section{Authors' contributions}

G-TL conceived the study. Y-HQ, LH, and G-FL carried out the experiments. $\mathrm{Y}-\mathrm{HQ}$ and ML performed bioinformatics analysis. Y-HQ and G-TL wrote the manuscript. All authors read and approved the final manuscript.

\section{Funding}

This work was supported by the 973 Program of the Ministry of Science and Technology of China (2015CB150601), the National Natural Science Foundation of China $(31371263 ; 31860021)$ and the Ba Gui Scholar Program of Guangxi Zhuang Autonomous Region of China (2014A002). The funding bodies had no role in the design of the study, collection, analysis, and interpretation of data and in writing the manuscript.

\section{Availability of data and materials}

All data generated or analyzed during this study are included in this published article.

\section{Ethics approval and consent to participate}

Not applicable.

\section{Consent for publication}

Not applicable.

\section{Competing interests}

The authors declare that they have no competing interests.

\section{Received: 7 May 2019 Accepted: 27 January 2020}

\section{Published online: 18 February 2020}

\section{References}

1. Ryan RP, Vorhölter FJ, Potnis N, Jones JB, Van Sluys MA, Bogdanove AJ, Dow JM. Pathogenomics of Xanthomonas: understanding bacterium-plant interactions. Nat Rev Microbiol. 2011;9(5):344-55.

2. Dow JM, Crossman L, Findlay K, He YQ, Feng JX, Tang JL. Biofilm dispersal in Xanthomonas campestris is controlled by cell-cell signaling and is required for full virulence to plants. PNAS. 2003;100:10995-1000.

3. Saul B, Bahar O, Parker JK, Fuente LD. Involvement of type IV pili in pathogenicity of plant pathogenic bacteria. Genes. 2011;2:706-35.

4. Slater H, Alvarez-Morales A, Barber CE, Daniels MJ, Dow JM. A twocomponent system involving an HD-GYP domain protein links cell-cell signalling to pathogenicity gene expression in Xanthomonas campestris. Mol Microbiol. 2000;38:986-1003.

5. Tang JL, Liu YN, Barber CE, Dow JM, Wootton JC, Daniels MJ. Genetic and molecular analysis of a cluster of rpf genes involved in positive regulation of synthesis of extracellular enzymes and polysaccharide in Xanthomonas campestris pathovar campestris. Mol Gen Genet. 1991;226:409-17.
6. Wilson TJ, Bertrand N, Tang JL, Feng JX, Pan MQ, Barber CE, Dow JM, Daniels MJ. The rpfA gene of Xanthomonas campestris pathovar campestris, which is involved in the regulation of pathogenicity factor production, encodes an aconitase. Mol Microbiol. 1998;28:961-70.

7. Zipfel C, Robatzek S, Navarro L, Oakeley EJ, Jones JDG, Felix G, Boller T. Bacterial disease resistance in Arabidopsis through flagellin perception. Nature. 2004;428:764-7.

8. He YW, Boon C, Zhou L, Zhang LH. Co-regulation of Xanthomonas campestris virulence by quorum sensing and a novel two-component regulatory system RavS/RavR. Mol Microbiol. 2009;71:1464-76.

9. Zhang SS, He YQ, Xu LM, Chen BW, Jiang BL, Liao J, Cao JR, Liu D, Huang YQ, Liang XX, Tang DD, Lu GT, Tang JL. A putative colR $x_{X 1049}$-Co/S XC1050 twocomponent signal transduction system in Xanthomonas campestris positively regulates $h r p C$ and hrpE operons and is involved in virulence, the hypersensitive response and tolerance to various stresses. Res Microbiol. 2008;159:569-78.

10. Li RF, Lu GT, Li L, Su HZ, Feng GF, Chen Y, He YQ, Jiang BL, Tang DJ, Tang $J$. Identification of a putative cognate sensor kinase for the twocomponent response regulator $\mathrm{HrpG}$, a key regulator controlling the expression of the hrp genes in Xanthomonas campestris pv. campestris. Environ Microbiol. 2014;16(7):2053-71.

11. Tao J, He CZ. Response regulator, VemR, positively regulates the virulence and adaptation of Xanthomonas campestris pv. campestris. FEMS Microbio Lett. 2010;304:20-8.

12. Qian W, Han ZJ, Tao J, He C. Genome-scale mutagenesis and phenotypic characterization of two-component signal transduction systems in Xanthomonas campestris pv. campestris ATCC 33913. Mol Plant Microbe In. 2008:21:1128-38.

13. Cui P, Li RF, Zhang DP, Tang JL, Lu GT. HpaP, a novel regulatory protein with ATPase and phosphatase activity, contributes to full virulence in Xanthomonas campestris pv. Campestris. Environ Microbiol. 2018;20(4): 1389-404.

14. Darzins A. The pilG gene product, required for Pseudomonas aeruginosa pilus production and twitching motility, is homologous to the enteric, single-domain response regulator CheY. J Bacteriol. 1993;175:5934-44.

15. Darzins A. Characterization of a Pseudomonas aeruginosa gene cluster involved in pilus biosynthesis and twitching motility: sequence similarity to the chemotaxis proteins of enterics and the gliding bacterium Myxococcus xanthus. Mol Microbiol. 1994;11:137-53.

16. Collins R, Saleem M, Derrick JP. Purification and three-dimensional Electron microscopy structure of the Neisseria meningitidis type IV Pilus biogenesis protein PilG. J Bacteriol. 2007;189:6389-96

17. Ryan RP, Fouhy Y, Lucey JF, Jiang BL, He YQ, Feng JX, Tang JL, Dow JM. Cyclic di-GMP signalling in the virulence and environmental adaptation of Xanthomonas campestris. Mol Microbiol. 2007:63(2):429-42.

18. Su HZ, Wu L, Qi YH, Liu GF, Lu GT and Tang JL. Characterization of the GntR family regulator HpaR1 of the crucifer black rot pathogen Xanthomonas campestris pathovar campestris. Sci Rep. 2015;6:19862.

19. Yang TC, Leu YW, Chang-Chien HC, Rouh-Mei H. Flagellar biogenesis of Xanthomonas campestris requires the alternative sigma factors RpoN2 and FliA and is temporally regulated by FlhA, FlhB, and FlgM. J Bacteriol. 2009; 191:2266-75.

20. He $Y Q$, Zhang $L$, Jiang $B L$, Zhang ZC, Xu RQ, Tang DJ, Qin J, Jiang W, Zhang X, Liao J, Cao JR, Zhang SS, Wei ML, Liang X, Lu GT, Feng JX, Chen B, Cheng J, Tang JL. Comparative and functional genomics reveals genetic diversity and determinants of host specificity among reference strains and a large collection of Chinese isolates of the phytopathogen Xanthomonas campestris pv.campestris. Genome Biol. 2007:8:218.

21. Bren $\mathrm{A}$, Eisenbach $\mathrm{M}$. The $\mathrm{N}$ terminus of the flagellar switch protein, FliM, is the binding domain for the chemotactic response regulator, CheY. J Mol Biol. 1998:278(3):507-14

22. Collin DM, Dahlquist FW. Switched or not?: the structure of Unphosphorylated CheY bound to the N terminus of FliM. J Bacteriol. 2006; 188(21):7354-63

23. Welch M, Oosawa K, Aizawa S-I, Eisenbach M. Phosphorylation-dependent binding of a signal molecule to the flagellar switch of bacteria. Proc Natl Acad Sci U S A. 1993:90:8787-91.

24. Welch M, Oosawa K, Aizawa S-I, Eisenbach M. Effects of phosphorylation, $\mathrm{Mg}^{2+}$, and conformation of the chemotaxis protein CheY on its binding to the flagellar switch protein FliM. Biochemistry. 1994;33:10470-6. 
25. An SQ, Febrer M, McCarthy Y, Tang DJ, Clissold L, Kaithakottil G, Swarbreck D, Tang JL, Rogers J, Dow JM, Ryan RP. High-resolution transcriptional analysis of the regulatory influence of cell-to-cell signalling reveals novel genes that contribute to Xanthomonas phytopathogenesis. Mol Microbiol. 2013;88(6):1058-69.

26. An S, Tang JL. Formation of a complex between HD-GYP, GGDEF and PilZ domain proteins regulates motility in Xanthomonas campestris. Microbiol Res. 2018. https://doi.org/10.4081/mr.2018.7601.

27. Zhang ZC, Zhao M, Xu LD, Niu XN, Qin HP, Li YM, Li ML, Jiang ZW, Yang X, Huang GH, Jiang W, Tang JL, He YQ. Genome-wide screening for novel candidate virulence related response regulator genes in Xanthomonas oryzae pv. oryzicola. Front Microbiol. 2018;9:1789.

28. Conrad JC. Physics of bacterial near-surface motility using flagella and type IV pili: implications for biofilm formation. Res Microbiol. 2012;163(9-10):619-29.

29. Yoshihara S, Geng X, Ikeuchi M. pilG gene cluster and split pill genes involved in pilus biogenesis, motility and genetic transformation in the cyanobacterium Synechocystis sp. PCC 6803. Plant Cell Physiol. 2002;43(5): 513-21.

30. Jenal U, Galperin MY. Single-domain response regulators: molecular switches with emerging roles in cell organization and dynamics. Curr Opin Microbiol. 2009;12(2):152-60.

31. Tammam S, Sampaleanu LM, Koo J, Sundaram P, Ayers M, Andrew Chong P, Forman-Kay JD, Burrows LL, Howe PL. Characterization of the PilN, PilO and PilP type IVa pilus subcomplex. Mol Microbiol. 2011;82(6):1496-514.

32. Hauberg L, Schmidt F, Scharf C, Dörr J, Völker U, Reinhold-Hurek B. Proteomic characterization of a pilR regulatory mutant of Azoarcus sp. strain BH72 with the aid of gel-based and gel-free approaches. Proteomics. 2010; 10(3):458-69.

33. Ryan RP, McCarthy Y, Kiely PA, O'Connor R, Farah CS, Armitage JP, Dow JM. Dynamic complex formation between HD-GYP, GGDEF and PilZ domain proteins regulates motility in Xanthomonas campestris. Mol Microbiol. 2012; 86:557-67.

34. Paul $\mathrm{K}$, Harmon JG, Blair DF. Mutational analysis of the flagellar rotor protein FliN: identification of surfaces important for flagellar assembly and switching. J Bacteriol. 2006;188:5240-8.

35. Lloyd SA, Whitby FG, Blair DF, Hill CP. Structure of the C-terminal domain of FliG, a component of the rotor in the bacterial flagellar motor. Nature. 1999; 400(6743):472-5

36. Yakushi T, Yang J, Fukuoka H, Homma M, Blair DF. Roles of charged residues of rotor and stator in flagellar rotation: comparative study using $\mathrm{H}^{+}$-driven and $\mathrm{Na}^{+}$- driven motors in Escherichia coli. J Bacteriol. 2006;188:1466-72.

37. Sarkar MK, Paul K, Blair D. Chemotaxis signaling protein CheY binds to the rotor protein FliN to control the direction of flagellar rotation in Escherichia coli. PNAS. 2010;107(20):9370-5.

38. Fulcher NB, Holliday PM, Klem E, Cann MJ, Wolfgang MC. The Pseudomonas aeruginosa Chp chemosensory system regulates intracellular CAMP levels by modulating adenylate cyclase activity. Mol Microbiol. 2010;76:889-904.

39. Daniels MJ, Barber CE, Turner PC, Sawczyc MK, Byrde RJW, Fielding AH. Cloning of genes involved in pathogenicity of Xanthomonas campestris pv. campestris using the broad host range cosmid pLAFR1. EMBO J. 1984;3: 3323-8.

40. Schäfer A, Tauch A, Jäger W, Kalinowski J, Thierbach G, Pühler A. Small mobilizable multi-purpose cloning vectors derived from the Escherichia coli plasmids pK18 and pK19: selection of defined deletions in the chromosome of Corynebacterium glutamicum. Gene. 1994;145:69-73.

41. Mazumder R, Phelps TJ, Krieg NR, Benoit RE. Determining chemotactic responses by two subsurface microaerophiles using a simplified capillary assay method. J Microbiol Methods. 1999;37:255-63.

42. Leng M, Lu ZJ, Qin ZS, Qi YH, Lu GT, Tang JL. Flp, a Fis-like protein, contributes to the regulation of type III secretion and virulence processes in the phytopathogen Xanthomonas campestris pv. Campestris. Mol Plant Pathol. 2019;20(8):1119-33.

\section{Publisher's Note}

Springer Nature remains neutral with regard to jurisdictional claims in published maps and institutional affiliations.

Ready to submit your research? Choose BMC and benefit from:

- fast, convenient online submission

- thorough peer review by experienced researchers in your field

- rapid publication on acceptance

- support for research data, including large and complex data types

- gold Open Access which fosters wider collaboration and increased citations

- maximum visibility for your research: over $100 \mathrm{M}$ website views per year

At BMC, research is always in progress.

Learn more biomedcentral.com/submissions 